Check for updates

Cite this: RSC Adv., 2019, 9, 13153

\title{
Elastic and hydrostatic behaviour of a zinc dietary supplement, zinc glycinate hydrate $\uparrow$
}

\author{
Muhammad Azeem, ${ }^{a}$ Muhammad Asif, ${ }^{b}$ Di Gui, ${ }^{a}$ Liyuan Dong, ${ }^{a}$ Chunlei Pei, ${ }^{\text {*c }}$ \\ Peixiang $\mathrm{Lu}^{\mathrm{ad}}$ and Wei $\mathrm{Li}(\mathbb{D}$ *ae
}

Coordination polymer based dietary supplement tablets are commonly consumed in our daily life and play an important role in the pharmaceutical industry. To fully understand their tableting process, their mechanical properties need to be comprehensively studied. In this work, the elastic and hydrostatic behaviour of a zinc supplement, zinc glycinate hydrate $\left(\mathrm{Zn}\left[\mathrm{O}_{2} \mathrm{CCH}_{2} \mathrm{NH}_{2}\right]_{2} \cdot \mathrm{H}_{2} \mathrm{O}\right)$, have been studied via density functional theory (DFT) calculations and high-pressure synchrotron powder X-ray diffraction. This material has a pseudo-layered structure and can be successfully exfoliated into nanosheets. The DFT calculated elastic moduli along the principal axes (13.84-36.11 GPa) indicate a significant elastic anisotropy of ZnG as expected for a layered system, and the directional dependent elastic modulus can be corroborated with the underlying atomic structure. In addition, the calculated B/G ratios (1.30-3.83) according to Pugh's criterion reveal that ZnG could be brittle under uniaxial stress ( $B$ and $G$ are bulk modulus and shear modulus, respectively). Furthermore, the measured $B$ is $\sim 31 \mathrm{GPa}$, which lies in the middle of the values between inorganic dietary supplements and small organic drug crystals. These results provide some quantitative information about the tableting process of the hybrid dietary supplement which could be different from their inorganic and organic pharmaceutical counterparts.

\author{
Received 16th January 2019 \\ Accepted 17th April 2019 \\ DOI: $10.1039 / c 9 r a 00385 a$ \\ rsc.li/rsc-advances
}

\section{Introduction}

Research on coordination polymers (CPs) has attracted immense attention in the past two decades due to their chemical diversity and combined properties of both organic and inorganic materials. ${ }^{1-3}$ CPs have been found to show promising applications in catalysis, ${ }^{4}$ gas storage and separation, ${ }^{5}$ ferromagnetism, ${ }^{6}$ and multiferroics. ${ }^{7}$ In addition to these applications, CPs can also serve as dietary supplements which have been widely consumed in our daily life to provide mineral nutrition. ${ }^{8}$ In terms of composition, CP based dietary supplements have better bioactivity and fewer side-effects than conventional purely inorganic food supplements, and play a more important role in the pharmaceutical industry. ${ }^{9}$ There are many CP based supplements, which include calcium (e.g. calcium gluconate, calcium aspartate),

${ }^{a}$ School of Physics, Huazhong University of Science and Technology, Wuhan 430074, China

${ }^{b}$ School of Chemistry and Chemical Engineering, Huazhong University of Science and Technology, Wuhan 430074, China

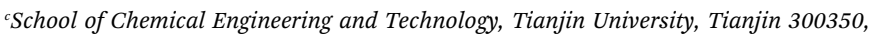
China.E-mail: chunlei.pei@tju.edu.cn

${ }^{d}$ Hubei Key Laboratory of Optical Information and Pattern Recognition, Wuhan Institute of Technology, Wuhan 430205, China

${ }^{e}$ School of Materials Science and Engineering, Nankai University, Tianjin 300350, China.E-mail:wl276@nankai.edu.cn

$\dagger$ Electronic supplementary information (ESI) available. See DOI: 10.1039/c9ra00385a zinc (e.g. zinc glycinate, zinc acetate), iron (e.g. ferrous fumarate), magnesium (e.g. magnesium malate), copper (e.g. copper glycinate), and potassium (e.g. potassium citrate) derivatives. ${ }^{10-12}$

Dietary supplements can be used in many dosage forms like powder, liquid, soft gel, capsule and tablet. Overall, the tablet form offers various advantages such as low cost, long term storage stability, good portability and easy usage. ${ }^{13}$ During tableting, these crystalline materials need to be milled and compacted, and the discrete elastic and plastic deformation in this complex process strongly determines the tabletability. ${ }^{8}$ For example, strong elastic recovery of food supplemental materials during compaction process could lead to detrimental capping and delamination. ${ }^{14}$ In addition, a better plasticity via slipping and motion of dislocation along some specific slipped planes, may result in more facile milling and better compactability. ${ }^{15}$ And the presence of water molecules in hydrated dietary supplements can also facilitate plastic deformation which could give tablets good strength and large volume reduction. ${ }^{16}$ In this context, a thorough understanding of the intrinsic elastic and plastic properties of these CPs based supplements at an atomic level is highly needed. However, there are very few studies concerning this important area, and the currently available knowledge is limited to a calcium system, calcium fumarate trihydrate. ${ }^{8}$ Considering the vast chemical and structural diversity of CPs based dietary supplements, other systems also need to be explored.

In the present work, we study the elastic and hydrostatic behaviour of a dietary supplement, zinc glycinate hydrate, ( $\mathrm{Zn}$ 
$\left[\mathrm{O}_{2} \mathrm{CCH}_{2} \mathrm{NH}_{2}\right]_{2} \cdot \mathrm{H}_{2} \mathrm{O}, \mathrm{ZnG}$ ) with a pseudo $2 \mathrm{D}$ structure through combined high-pressure powder X-ray diffraction (HP-PXRD) experiments and first principles calculations. Our results demonstrate the intrinsic relationship between the underlying crystal structure and hydrostatic behaviour and elasticity of this hybrid dietary supplement. Moreover, we found that the strong mechanical anisotropy of this $2 \mathrm{D}$ hybrid supplement results in its easy delamination into nanosheets under shearing stress which need to be taken into account during tableting.

\section{Experimental}

\subsection{Synthesis}

All chemicals and solvents purchased as reagent grade and were used as received without further purification. Typically, glycine $(0.23 \mathrm{~g}, 3 \mathrm{mmol})$ was dissolved in $10 \mathrm{~mL}$ of water and heated up to $75{ }^{\circ} \mathrm{C}$. Meanwhile, zinc hydroxide $(0.15 \mathrm{~g}, 1.5 \mathrm{mmol})$ was dissolved in $10 \mathrm{~mL}$ of water in another beaker at room temperature. Both solutions were mixed and placed under refrigeration overnight. The solution was taken and filtrate was mixed with $10 \mathrm{~mL}$ of absolute ethanol and placed the mixture in a fume hood for slow evaporation at room temperature. Colorless plates of ZnG single crystals were obtained and washed with chilled ethanol.

\subsection{Preparation of nanosheets}

The nanosheets were prepared by mixing $0.1 \mathrm{mg}$ (ZnG powder) in $7 \mathrm{~mL}$ (ethanol) in a $10 \mathrm{~mL}$ cylindrical vial and sonicated for about $20 \mathrm{~min}$ in a KQ3200DE ultrasonic cleaner equipment with fixed power $(120 \mathrm{~W})$ and frequency $(40 \mathrm{kHz})$. The mild milky suspension in the vial was centrifuged at a rate of $5000 \mathrm{rpm}$ for 5 minutes.

\subsection{Atomic force microscopy}

A Digital Instrument Multimode SPM in the tapping mode with BRUKER RTESPA-300 tip was used for observing the surface topographies and the corresponding thickness of different exfoliated nanosheets, ${ }^{17}$ deposited on a clean silicon substrate $\left(1 \times 1 \mathrm{~cm}^{2}\right)$ by drop casting method. The images were taken at a resolution of $256 \times 256$ pixel, by scanning an area of $10 \times 10$ $\mu \mathrm{m}^{2}$ at a rate of $1.00 \mathrm{~Hz}$. Besides that, the values of operation point, integral gain, and P gain were fixed at $0.153 \mathrm{~V}, 1500$ and 0.001 , respectively.

\subsection{Transmission electron microscopy}

Bright field images of as prepared nanosheets dropped on a holy carbon coated copper grids were collected with Titan 60$300 \mathrm{Cs}$, corrected transmission electron microscope operating at $300 \mathrm{kV}$, while selected area electron diffraction (SAED) patterns were obtained by JEM-2100 electron microscope operating at $200 \mathrm{kV}$.

\subsection{Scanning electron microscopy}

Scanned images of as prepared nanosheets on silicon substrates were collected with the scanning electron microscope Quanta 200, operating at $30 \mathrm{kV}$.

\subsection{High-pressure synchrotron powder X-ray diffraction}

A diamond anvil cell (DAC) with a culet diameter of $400 \mu \mathrm{m}$ and a stainless-steel gasket of $40 \mu \mathrm{m}$ thickness were used. A synchrotron radiation $(\lambda=0.6199 \AA)$ was focused to a $36 \times 12$ $\mu \mathrm{m}^{2}$ spot by means of Kirkpatrick-Baez mirrors to collect data by Pilatus $2 \mathrm{M}$ detector integrated via a FIT2D suit of package, ${ }^{18}$ on $4 \mathrm{~W} 2$ beamline at Beijing Synchrotron Radiation Facility (BSRF) at different pressures. ${ }^{19}$ A well ground powder sample was loaded in a hole of above mentioned stainless-steel gasket (200 $\mu \mathrm{m}$ of diameter) with few ruby balls. The ruby chips were used for pressure calibration, while silicon oil as a hydrostatic medium in DAC. The pressure was determined by ruby fluorescence inside DAC with an error of $0.1 \mathrm{GPa} .{ }^{20}$ The unit cell parameters for different pressures were refined by the Le Bail method.

\subsection{First-principles calculation}

The first principles calculations were made to obtain the elastic anisotropy of material by using CASTEP, ${ }^{21}$ a total energy package based on plane-wave pseudo-potential density functional theory. ${ }^{22}$ The functions developed in local density approximation (LDA), ${ }^{23,24}$ were used to indicate the exchangecorrelation energy. Optimized ultrasoft pseudo-potentials, ${ }^{25}$ were adopted to model the effective interaction between the valence electrons and atomic cores, which allowed us to use a set of relatively small plane-wave basis without compromising the computational accuracy. The kinetic energy cut-off was set as $400 \mathrm{eV}$ and Monkhorst-Pack, ${ }^{26} k$-point mesh spanning was less than $0.04 \AA^{-1}$.

\section{Results and discussion}

\subsection{Crystal structure}

ZnG crystalizes in the monoclinic crystal system with space group $C 2 / c$ as reported previously. ${ }^{27}$ There are two crystallographic independent zinc atoms ( $\mathrm{Zn} 1$ and $\mathrm{Zn} 2$ ) which are chelated by two different glycinates in the asymmetric unit. Interestingly, the two chelating glycinate rings around both zinc atoms show almost perpendicular orientations. The $\mathrm{O} 6$ of $\mathrm{Zn} 2$ and $\mathrm{O} 4$ of $\mathrm{Zn} 1$ bound to $\mathrm{Zn} 1$ and $\mathrm{Zn} 2$, respectively, to form a zinc tetramer with pending $\mathrm{O} 2$ and $\mathrm{O} 8$ from carboxylate groups $\mathrm{C} 2$ and $\mathrm{C} 8$, and adjacent tetramers link each other to form a one-dimensional (1D) polymer chain approximately along the $\langle 410\rangle$ direction. These 1D polymer chains deviate $26.67(1)^{\circ}$ and $63.33(1)^{\circ}$ from the $a$ - and $b$-axis, respectively. In addition, there are also three water molecules at different positions in the asymmetric unit, namely O9, O10 and 011. These three water molecules, amino and carboxylate groups of the two glycinate ligands form abundant hydrogen bonding which connect the 1D chains along other two orthogonal directions. Strikingly, there are significantly more hydrogen bonding connections within the $a b$-plane but much less along the $c$-axis, making this compound a pseudo 2D system in terms of hydrogen bonding strength. The layered arrangement and hydrogen bonding modes in the $a b$-plane are displayed in Fig. 1a, and adjacent layers of tetramers are stacked along the $c$ - 
axis with a layer thickness of $\sim 0.746 \mathrm{~nm}$ (Fig. 1b). The arrangements of hydrogen bonding along other directions are shown in Fig. S1a and b. $\dagger$

\subsection{Physical exfoliation and characterizations}

The pseudo 2D nature of ZnG crystal suggests that it could be exfoliated into nanosheets as expected for a 2D material. Accordingly, the ZnG crystals were ground into powder and sonicated in ethanol for about $20 \mathrm{~min}$ to obtain nanosheets. The typical surface morphology and thickness of as-prepared nanosheets measured by AFM are shown in Fig. 2. The nanosheets stacking like overlapped snowflakes are displayed on Fig. 2a, which have different lateral dimensions at the micron size. The stepwise nanosheets pointed in Fig. 2a exhibit different heights of nanosheets, which is commonly expected from liquid-assisted exfoliation correspond to 4-, 6-, 7-, and 12layers thickness. However, nanosheets from other scans exhibit same thicknesses with a uniform height difference about $\sim 4.2 \mathrm{~nm}$ (Fig. $\mathrm{S} 2 \dagger$ ). The AFM characterisations give clear evidence that $\mathrm{ZnG}$ has a typical behaviour of $2 \mathrm{D}$ materials.

The morphology, crystallinity of exfoliated nanosheets were also examined by TEM characterization. The bright field image of stacked nanosheets is shown in Fig. 2c which show that the thickness and lateral sizes of these nanosheets are different in the dark and bright areas. This variation in morphology of different areas may be attributed to incomplete delamination or restacking of exfoliated nanoflakes. ${ }^{28,29}$ Like other CPs and metal-organic frameworks (MOFs), ZnG nanosheets are also very sensitive to the electron beam, which leads to the damages (holes) on the surface of nanosheets seen in Fig. 2c. The selected area electron diffraction (SAED) patterns were collected to examine the crystallinity of the exfoliated nanosheets, and the diffracted spots shown in Fig. 2d correspond to the (800), (440) and equivalent planes.

SEM images show stacking feature of exfoliated nanosheets. The lateral sizes are different at various points showing the irregular breakdown of nanosheets during exfoliation under the application of mechanical forces by sonicator (Fig. 3).

\subsection{Elastic properties}

To fully understand the layered structural nature of ZnG, its full elastic constants were calculated using the density functional

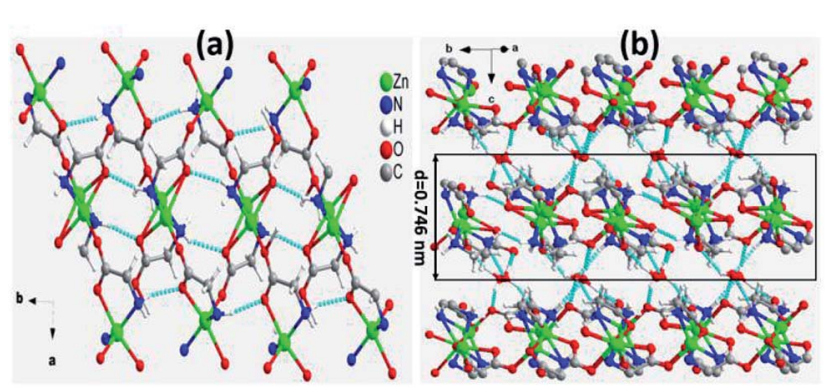

Fig. 1 Crystal structure of ZnG. (a) Two dimensional layered arrangement in the $a b$-plane. (b) Layer stacking pattern along the $c$ axis. Color scheme: $\mathrm{Zn}$, light green; $\mathrm{O}$, red; $\mathrm{C}$, grey; $\mathrm{N}$, blue and $\mathrm{H}$, white.
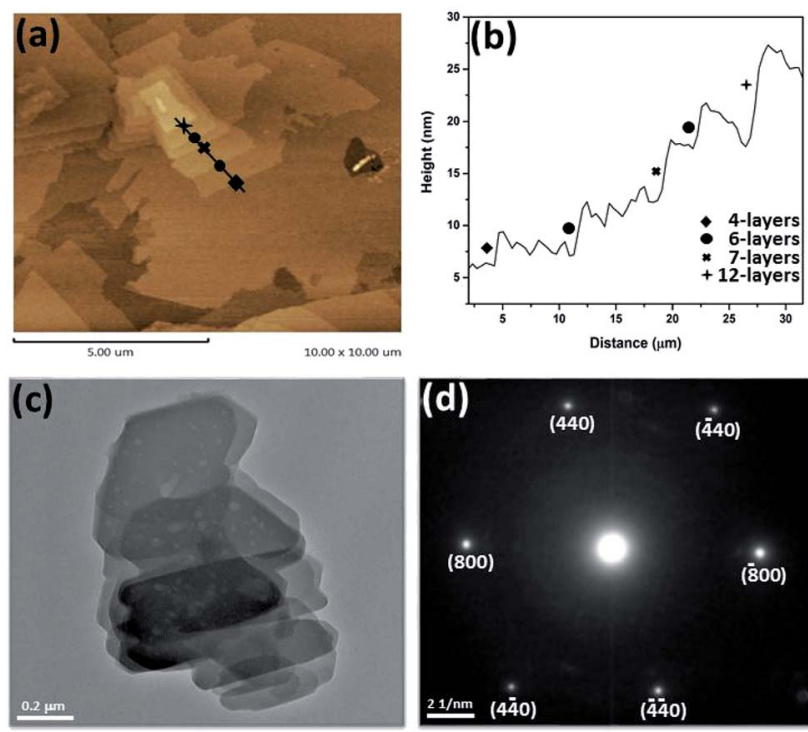

Fig. 2 AFM surface topographies and corresponding height and TEM images. Where (a) step wise stacking of nanosheets. (b) Graphical representation of thickness, $\sim 3.1, \sim 4.6, \sim 5.4$, and $\sim 9.2 \mathrm{~nm}$ from the bottom layer correspond to 4-, 6-, 7-, and 12-layers thickness with different symbolic indicators. (c) Bright field image of exfoliated nanosheets by TEM with holes. (d) SAED patterns of layered nanosheets.

theory (DFT) through the CASTEP codes. The calculated 13 independent elastic constants are listed in Table S1. $\dagger$ The elastic moduli $(E)$, shear moduli $(G)$ and Poisson's ratios $(\nu)$ were then extracted from the DFT calculation results (Table S2 $\dagger$ ). The 3 D view of $E$ and its projection normal to the (100) plane are displayed in Fig. $4 \mathrm{a}$ and b. Clearly, the $a$-axis is the stiffest direction, while the $c$-axis is the most compliant axis. In the $3 \mathrm{D}$ view, $E$ along different orientations is represented by an irregular sphere with elliptical corners, which indicates the significant anisotropy of ZnG crystal. The yellow, light blue and green corners pointing to the [100], approximate [010] and [001] direction, respectively. As shown in the $2 \mathrm{D}$ view, $E_{[100]}$ and $E_{\sim[001]}$ are 36.11 and $13.84 \mathrm{GPa}$, respectively. In addition, $E_{\sim[010]}$ is $30.61 \mathrm{GPa}$, which is very close to $E_{[100]}$ but is more than twice of $E_{\sim[001]}$. Clearly, ZnG is significantly compressible along the $c$ axis but much stiffer along the $a$ - and $b$-axis, which is expected for this pseudo-layered system and consistent with the observation of successful exfoliation.
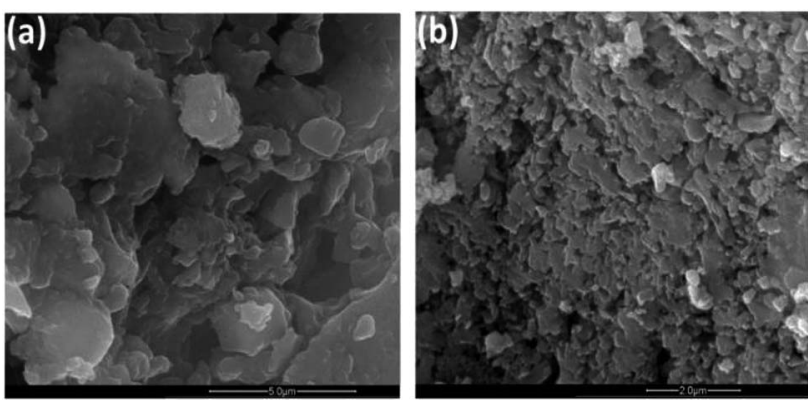

Fig. 3 SEM images of ZnG nanosheets at different magnification (a and b). 

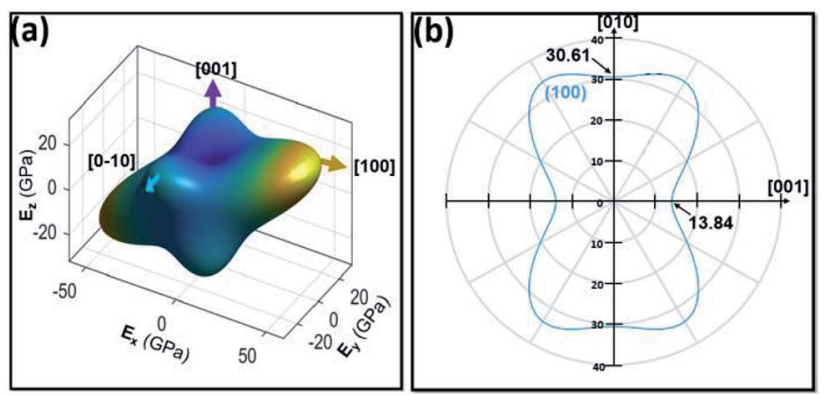

Fig. 4 Surface counters of direction depended elastic moduli: (a) 3D view; (b) 2D view projected normal to the (100) plane.

The elastic anisotropy of the layered structure, defined as the ratio $A_{\mathrm{E}}=E_{[100]} / E_{\sim[001]}$ is about 2.61 . This $A_{\mathrm{E}}$ value is about an order of magnitude lower than graphite, and about $50 \%$ of those values from those of h-BN, $\mathrm{MoS}_{2}, \mathrm{WS}_{2}$, black $\mathrm{P}$ and a layered hybrid perovskite [(benzylammonium $\left.)_{2} \mathrm{PbBr}_{4}\right]^{30}$ Accordingly, the low $A_{\mathrm{E}}$ of $\mathrm{ZnG}$ gives rise to a lower ease level of exfoliation, ${ }^{31}$ as observed from AFM and TEM experiments. Furthermore, the difference in $E_{(100)}$ and $E_{(010)}$ can be explained by the underlying structural arrangements within the pseudo2D layer. Specifically, the $1 \mathrm{D}$ ZnG polymer chains are $26.67(1)^{\circ}$ and $63.33(1)^{\circ}$ with respect to the $a$ - and $b$-axis, which gives rise to more projection of the rigid 1D chains along [100] rather than [010], hence higher rigidity along [100] and more compliance along [010].

Compared with other types of dietary supplements, the $E$ values of $\mathrm{ZnG}$ is very close to the values of hybrid dietary supplement, calcium fumarate trihydrate, and inorganic dietary supplement, $\mathrm{MgO}$ (Table 1). However, these $E$ values are substantially lower than those from $\mathrm{CaCO}_{3}$ and much higher than those of zinc acetate dehydrate and small molecule pharmaceutical crystals.

The 3D and 2D contours of Poisson's ratio $(\nu)$ of $\mathrm{ZnG}$ are displayed in Fig. 5, which show the Poisson's ratios are between 0.01 to 0.76 . These values are narrower than those of graphene (0.99 to 0.01$)$ and broader than h-BN $(0.49$ to 0.06$)$, but approximate to those from [(benzylammonium) $\left.{ }_{2} \mathrm{PbBr}_{4}\right]$ (0.87 to $0.01) .{ }^{30}$ It is well known that the smaller the Poisson's ratio is, the more brittle the material is. ${ }^{32}$ A solid is usually defined as brittle when its Poisson's ratios lower than 0.26. For ZnG crystal, it has many directions which possess Poisson's ratios smaller than this critical value, hence implying that it could be brittle in nature under uniaxial stretching or compressing. We also calculated the values of shear modulus $(G)(6.52-19.15 \mathrm{GPa})$ and corresponding values of $B / G(1.30-3.83)$. According to the Pugh's criteria, materials with $B / G<1.75$ are brittle. In this regard, ZnG would be brittle. ${ }^{33}$ Nevertheless, this brittle feature of $\mathrm{ZnG}$ would facilitate its milling during the tableting process. The different uniaxial elastic properties of $\mathrm{ZnG}(E$ and $\nu)$ indicate that its tableting would be in principle different from those organic drug crystals and inorganic food supplements.

\subsection{Hydrostatic behaviour}

To explain the hydrostatic behaviour of ZnG single crystal under pressure, we performed HP-PXRD experiments at ambient temperature. The collected powder patterns were refined using the Le Bail method to obtain lattice parameters at different pressures (Table $\mathrm{S} 7 \dagger$ ). The relative changes in lattice parameters $(a, b, c, V$ and $\beta$ ) are shown in Fig. 6a-d from ambient to $12.10 \mathrm{GPa}$. The percentage decrease in $a, b, c$, and $V$ are $4.81 \%$, $5.39 \%, 11.94 \%$ and $20.81 \%$, respectively, which shows a normal compressing behaviour under pressure. This normal behaviour could be perceived in the Bragg peak shifting from a lower to a higher angle (Fig. 7a and b). Eventually, the ZnG unit cell shrinks more along the $c$-axis (stacked-layers direction) as interlayer spacing distance decreases rapidly with increasing pressure, followed by the $b$-axis, and the least compressibility is along the $a$-axis.

Consequently, from the HP-PXRD and DFT calculations we can conclude that material shows mechanical anisotropic

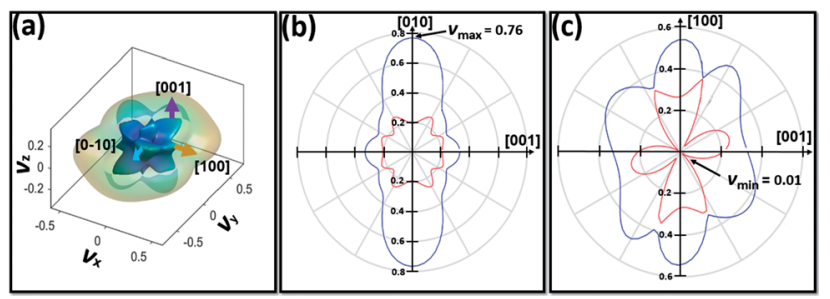

Fig. 5 Surface counters of direction depended Poisson's ratios: (a) 3D view; (b) 2D view projected normal to the (100) plane. (c) 2D view projected normal to the (010) plane.

Table 1 Elastic and bulk moduli of some reported small molecule drug crystals and dietary supplement compounds

\begin{tabular}{|c|c|c|c|c|}
\hline Category & Material & $E(\mathrm{GPa})$ & $K_{\mathrm{o}}(\mathrm{GPa})$ & Ref. \\
\hline \multirow[t]{2}{*}{ Inorganic } & $\mathrm{CaCO}_{3}$ & $\sim 80$ & $\sim 73$ & 35 \\
\hline & $\mathrm{MgO}$ & $\sim 300$ & $\sim 160$ & 36 \\
\hline & Piracetam (form V) & Not reported & $\sim 13$ & 38 \\
\hline & DL-Mandelic acid (form II) & Not reported & $\sim 13$ & 39 \\
\hline & Theophylline-methyl gallate & $\sim 15$ & Not reported & 15 \\
\hline & Zinc acetate dihydrate & $\sim 4.3-5.8$ & $\sim 20$ & 17 \\
\hline & ZnG & $\sim 14$ (out-of-plane), $\sim 31-36$ (in-plane) & $\sim 31$ & This work \\
\hline
\end{tabular}




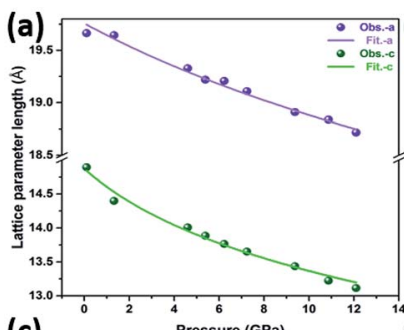

(c)

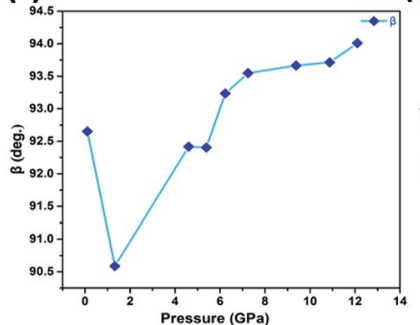

(d)
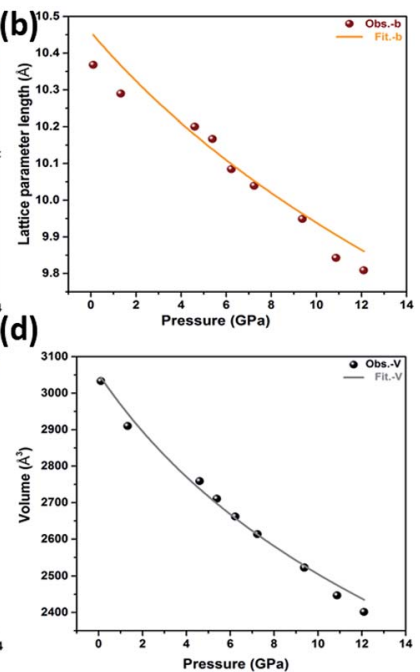

Fig. 6 Relative changes in lattice parameters of $\mathrm{ZnG}$ unit cell as a function of pressure. (a) Relative changes in $a$ and $c$, (b) in $b$, (c) in $\beta$. (d) Evolution of unit cell volume as a function of pressure. The solid lines in (a), (b) and (d) indicate the fits using the second-order BirchMurnaghan equation of state.

behaviour under pressure ( 0 to $12.10 \mathrm{GPa}$ ) without undergoing any phase transition (Fig. 7). Pressure-induced phase transformation can potentially hinder the tableting process, because it can cause instability and ineffectiveness in pharmaceutical materials. ${ }^{16}$ Therefore, this virtue could be advantageous to the pharmaceutical formulation of ZnG.

The bulk modulus $\left(K_{\mathrm{o}}\right)$ of $\mathrm{ZnG}$ was calculated using the second-order Birch-Murnaghan equation of state (EoS) through the PASCal software. ${ }^{34}$ The resultant value of $K_{\mathrm{o}}$ is $31 \pm 2 \mathrm{GPa}$, which is reasonably close to the obtained value through DFT calculations ( $25 \pm 2 \mathrm{GPa}$ ). The $K_{\mathrm{o}}$ of ZnG indicates its higher resistance to hydrostatic compression compared to some small molecule pharmaceutical materials and CPs-based dietary supplements (Table 1), which could affect the compactability of ZnG powder in the tableting process. However, this value is substantially smaller than those from inorganic dietary supplements, implying a more facile compaction attributed to its hybrid nature. The relative changes calculated by PASCal software for principal axes and corresponding linear compressibility along different directions are observed in the indicatrix shown in Fig. $\mathrm{S} 5, \dagger$ in which the principal axis $X_{1}, X_{2}$
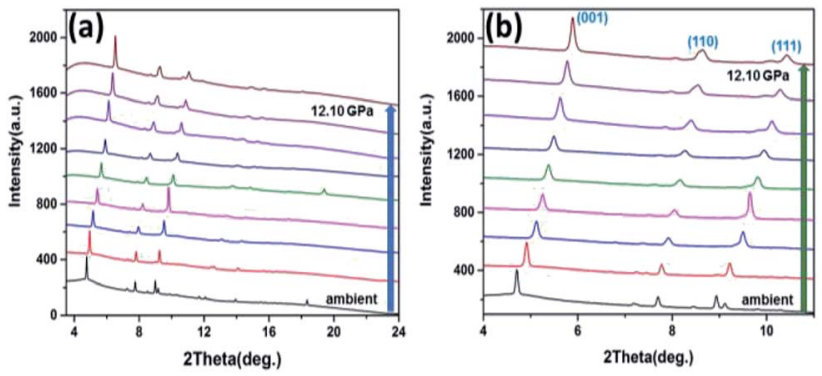

Fig. 7 HP-PXRD patterns at different pressure points. (a) Shifting of peaks from lower to a higher angle for all pressures. (b) Enhanced Bragg peaks of (001), (110) and (111). and $X_{3}$ are along the $a$-, approximately $b$ - and $c$-axis, respectively. The least linear compressibility is along $X_{1}\left(K_{X_{1}}=3.79 \mathrm{TPa}^{-1}\right)$, followed by $X_{2}\left(K_{X_{2}}=4.75 \mathrm{TPa}^{-1}\right)$, and finally the largest along $X_{3}$ $\left(K_{X_{3}}=9.11 \mathrm{TPa}^{-1}\right)$.

According to our DFT calculations, the values of Young's modulus along [100], [010] and [001] are 36.11, 30.61 and 13.84 GPa, respectively. These results show that ZnG can be compressed easily along [001] direction but hard along [100]. The behaviour can be observed in high-pressure synchrotron powder X-ray diffraction measurements in which reduction in lattice parameter ' $a$ ' (along [100] direction) is the smallest $(4.81 \%)$ as compared to the values $(5.39 \%$ and $11.94 \%)$ for lattice parameters ' $b$ ' and ' $c$ ' (along $\sim[010]$ and [001] directions, respectively). Similarly, the calculated compressibilities using PASCal software, based on experimental data show the same trend. These values change as; compressibility along $X_{1}$ (approximately along $a$-axis) < compressibility along $X_{2}$ (along $b$ axis) < compressibility along $X_{3}$ (approximately along $c$-axis), which is in reasonable agreement with our DFT calculations.

\section{Conclusions}

The physical exfoliation, elastic and hydrostatic behaviour of zinc glycinate hydrate, $\mathrm{Zn}\left[\mathrm{O}_{2} \mathrm{CCH}_{2} \mathrm{NH}_{2}\right]_{2} \cdot \mathrm{H}_{2} \mathrm{O}$, have been comprehensively explored via microscopic characterizations, DFT calculations and HP-PXRD. Interestingly, this 1D coordination polymer has a pseudo-2D feature and can be successfully exfoliated into nanosheets like other $2 \mathrm{D}$ crystals. Such a 2D nature of ZnG particles could limit the powder flowability, which might be detrimental to the die filling process and the following compaction. Moreover, the small $B / G$ values and the existence of abundant hydrogen-bonding in $\mathrm{ZnG}$ indicate that it could be brittle upon compression. Furthermore, the HP-PXRD experiments reveal that there is no phase transition up to 12.10 GPa, which could exclude any structural changes and associated function alterations during milling and compaction in the pharmaceutical process. Based on the above quantitative findings, we envision that the tableting process should be adapted for manufacturing hybrid dietary supplements compared with their inorganic and organic pharmaceutical counterparts.

\section{Conflicts of interest}

There are no conflicts to declare.

\section{Acknowledgements}

We acknowledge financial support from the National Natural Science Foundation of China (Grant No. 21571072) and the Fundamental Research Funds for the Central Universities (Nankai University, No. 63196006). The work concerning in situ HP-PXRD measurements were performed at beamline $4 \mathrm{~W} 2$, Beijing Synchrotron Radiation Facility (BSRF). We also thank the Analytical and Testing Center in Huazhong University of Science and Technology (HUST) for technical support. Special 
thanks to Dr Zhao Lu at Analytical and Testing Center of HUST for the guidance of TEM characterizations.

\section{Notes and references}

1 C. N. R. Rao, A. K. Cheetham and A. Thirumurugan, J. Phys.: Condens. Matter, 2008, 20, 083202.

2 A. K. Cheetham and C. Rao, Science, 2007, 318, 58-59.

3 A. K. Cheetham, C. N. R. Rao and R. K. Feller, Chem. Commun., 2006, 4780-4795.

4 P. Horcajada, R. Gref, T. Baati, P. K. Allan, G. Maurin, P. Couvreur, G. Ferey, R. E. Morris and C. Serre, Chem. Rev., 2011, 112, 1232-1268.

5 J. R. Li, R. J. Kuppler and H. C. Zhou, Chem. Soc. Rev., 2009, 38, 1477-1504.

6 D. F. Weng, Z. M. Wang and S. Gao, Chem. Soc. Rev., 2011, 40, 3157-3181.

7 P. Jain, V. Ramachandran, R. J. Clark, H. D. Zhou, B. H. Toby, N. S. Dalal, H. W. Kroto and A. K. Cheetham, J. Am. Chem. Soc., 2009, 131, 13625-13627.

8 S. Sun, S. Henke, M. T. Wharmby, H. H. M. Yeung, W. Li and A. K. Cheetham, Inorg. Chem., 2015, 54, 11186-11192.

9 L. García-Rico, J. Leyva-Perez and M. E. Jara-Marini, Food Chem. Toxicol., 2007, 45, 1599-1605.

10 D. A. Straub, Nutr. Clin. Pract., 2007, 22, 286-296.

11 H. Jesserer, Dtsch. Med. Wochenschr., 1967, 92, 1776-1777.

12 J. C. Larsen, K. K. Norby, P. Lund and V. M. Beltoft, EFSA J., 2008, EFSA-Q-2008-059.

13 L. Han, J. Elliott, A. Bentham, A. Mills, G. Amidon and B. Hancock, Int. J. Solids Struct., 2008, 45, 3088-3106.

14 V. Mazel, V. Busignies, H. Diarra and P. Tchoreloff, J. Pharm. Sci., 2013, 102, 4009-4014.

15 S. Chattoraj, L. Shi and C. C. Sun, CrystEngComm, 2010, 12, 2466-2472.

16 S. Varughese, M. S. Kiran, U. Ramamurty and G. R. Desiraju, Angew. Chem., Int. Ed. Engl., 2013, 52, 2701-2712.

17 G. Feng, W.-X. Zhang, L. Dong, W. Li, W. Cai, W. Wei, L.-J. Ji, Z. Lin and P. Lu, Chem. Sci., 2019, 10, 1309-1315.

18 J. Hammersley, FIT2D V12.012 Reference Manual, ESRF, Grenoble, France, 1996.

19 D. Gui, L.-J. Ji, A. Muhammad, W. Li, W.-Z. Cai, Y.-C. Li, X.-D. Li, X. Wu and P.-X. Lu, J. Phys. Chem. Lett., 2018, 9, 751-755.

20 G. J. Piermarini, S. Block, J. Barnett and R. Forman, J. Appl. Phys., 1975, 46, 2774-2780.

21 S. J. Clark, M. D. Segall, C. J. Pickard, P. J. Hasnip, M. I. Probert, K. Refson and M. C. Payne, Z. Kristallogr. Cryst. Mater., 2005, 220, 567-570.
22 M. Payne, Rev. Mod. Phys., 1992, 64, 1045.

23 J. P. Perdew and A. Zunger, Phys. Rev. B: Condens. Matter Mater. Phys., 1981, 23, 5048-5079.

24 D. M. Ceperley and B. Alder, Phys. Rev. Lett., 1980, 45, 566.

25 A. M. Rappe, K. M. Rabe, E. Kaxiras and J. D. Joannopoulos, Phys. Rev. B: Condens. Matter Mater. Phys., 1990, 41, 12271230.

26 H. J. Monkhorst and J. D. Pack, Phys. Rev. B: Solid State, 1976, 13, 5188.

27 S. Konar, K. Gagnon, A. Clearfield, C. Thompson, J. Hartle, C. Ericson and C. Nelson, J. Coord. Chem., 2010, 63, 33353347.

28 L. Dou, A. B. Wong, Y. Yu, M. Lai, N. Kornienko, S. W. Eaton, A. Fu, C. G. Bischak, J. Ma and T. Ding, Science, 2015, 349, 1518-1521.

29 Y. Peng, Y. Li, Y. Ban, H. Jin, W. Jiao, X. Liu and W. Yang, Science, 2014, 346, 1356-1359.

30 G. Feng, Y. Qin, C. Ran, L.-J. Ji, L. Dong and W. Li, APL Mater., 2018, 6, 114201.

31 L.-J. Ji, Y. Qin, D. Gui, W. Li, Y. Li, X. Li and P. Lu, Chem. Mater., 2018, 30(24), 8732-8738.

32 L.-Y. Dong, S.-J. Sun, Z.-Y. Deng, W. Li, F.-X. Wei, Y.-J. Qi, Y.-C. Li, X.-D. Li, P.-X. Lu and U. Ramamurty, Comput. Mater. Sci., 2018, 141, 49-58.

33 S. F. Pugh, London, Edinburgh Dublin Philos. Mag. J. Sci., 2009, 45, 823-843.

34 M. J. Cliffe and A. L. Goodwin, J. Appl. Crystallogr., 2012, 45, 1321-1329.

35 C. Merkel, J. Deuschle, E. Griesshaber, S. Enders, E. Steinhauser, R. Hochleitner, U. Brand and W. W. Schmahl, J. Struct. Biol., 2009, 168, 396-408.

36 D. Cáceres, I. Vergara, R. González and Y. Chen, Philos. Mag. A, 2002, 82, 1159-1171.

37 I. D. H. Oswald, A. R. Lennie, C. R. Pulham and K. Shankland, CrystEngComm, 2010, 12, 2533-2540.

38 F. P. Fabbiani, D. R. Allan, W. I. David, A. J. Davidson, A. R. Lennie, S. Parsons, C. R. Pulham and J. E. Warren, Cryst. Growth Des., 2007, 7, 1115-1124.

39 W. Cai, J. d. Marciniak, M. Andrzejewski and A. Katrusiak, J. Phys. Chem. C, 2013, 117, 7279-7285.

40 Y. Kim, K. Machida, T. Taga and K. Osaki, Chem. Pharm. Bull., 1985, 33, 2641-2647.

41 S. Varughese, M. S. R. N. Kiran, K. A. Solanko, A. D. Bond, U. Ramamurty and G. R. Desiraju, Chem. Sci., 2011, 2, 2236. 